This section features a recent systematic review that is indexed on PEDro, the Physiotherapy Evidence Database (http://www.pedro. org.au). PEDro is a free, web-based database of evidence relevant to physiotherapy.

\section{Walking exercise for chronic musculoskeletal pain (PEDro synthesis)}

- O'Connor SR, Tully MA, Ryan B, et al. Walking exercise for chronic musculoskeletal pain: systematic review and meta-analysis. Arch Phys Med Rehabil 2015;96:724-34.

\section{BACKGROUND}

Chronic musculoskeletal pain (CMP) is a major cause of morbidity. ${ }^{1}$ Aerobic exercise is recommended for people with CMP to reduce pain and increase functional status. ${ }^{2}{ }^{3}$ Walking is associated with a low risk of musculoskeletal injury, ${ }^{4}$ is safe for previously sedentary individuals ${ }^{5}$ and improves cardiovascular comorbidities. $^{6-8}$

\section{AIM}

The aim of this systematic review was to examine the effects of walking interventions on pain and self-reported function in adults with CMP.

\section{SEARCHES AND INCLUSION CRITERIA}

Six databases were searched (MEDLINE, Cochrane, CINAHL, PsychINFO, Sport Discus and PEDro) using a combination of terms including 'walking', 'aerobic exercise', 'musculoskeletal pain', 'low back pain', 'arthritis' and 'fibromyalgia'. Eligible studies were randomised controlled trials (RCTs) or quasi-RCTs including adults with a diagnosis of chronic low back pain, osteoarthritis or fibromyalgia syndrome.

\section{INTERVENTION}

Studies including land-based or treadmill-based walking interventions were considered eligible. Studies were required to include as comparator a non-exercise or non-walking exercise control.

\section{MAIN OUTCOMES}

Primary outcomes were pain and self-reported function.

\section{STATISTICAL METHODS}

Internal and external validity of included studies was rated as 'good', 'fair', or 'poor', using predefined criteria (US Preventive Services Task Force (USPSTF) method). Studies were considered clinically homogeneous based on similarities in patient demographic characteristics and intervention methods. Statistical heterogeneity was analysed using the $\chi^{2}$ test and $\mathrm{I}^{2}$ test statistics. Random-effects models for inverse variance were adopted to calculate the pooled mean difference (MD) and $95 \%$ CI. Duration of follow-up was categorised as short term ( $\leq 8$ weeks), medium term (2-12 months), or long term (>12 months).

\section{RESULTS}

Literature searches retrieved 2760 records. Of these, 26 studies met the inclusion criteria for this review. Seventeen studies were suitable for meta-analysis. In most studies (73\%), walking programmes were supervised, such as in a hospital clinic or gymnasium. Among home-based programmes, three studies used pedometers to assist with walking goals and three others used time-based walking-goals. Mean duration of follow-up assessment was $1.8 \pm 0.4$ months for studies with short-term outcomes, $4.9 \pm 1.9$ months for studies with medium-term outcomes and $18.4 \pm 7.6$ months for studies with long-term outcomes.

Five studies met all criteria for internal validity and nine studies met all criteria for external validity, and were rated as 'good'. Fifteen studies were rated as 'fair' for internal validity and seventeen were rated as 'fair' for external validity. The remaining five studies were rated as 'poor' for internal validity. The interventions in 10 studies (38\%) met all the American College of Sports Medicine guidelines for the quantity and quality of aerobic exercise.

Meta-analysis revealed that, in comparison with non-exercise or non-walking exercise control, walking interventions significantly decreased pain at short term $(\mathrm{MD}=-5.31 ; 95 \% \mathrm{CI}-8.06$ to -2.56$)$ and medium term $(\mathrm{MD}=-7.92 ; 95 \% \mathrm{CI}-12.37$ to $-3.48)$, but no effect for long term was observed $(\mathrm{MD}=-2.22$; $95 \%$ CI -6.03 to 1.59 ). For self-reported function, significant differences for all time points favouring walking interventions (short-term $\mathrm{MD}=-6.47 ; 95 \% \mathrm{CI}-12.00$ to -0.95 ; mediumterm $\mathrm{MD}=-9.31 ; 95 \% \mathrm{CI}-14.00$ to -4.61 ; and long-term $\mathrm{MD}=-5.22 ; 95 \% \mathrm{CI}-7.21$ to -3.23 ) were found. Sensitivity analyses excluding studies in which walking was combined with a cointervention did not alter overall results.

\section{CONSIDERATIONS/LIMITATIONS}

Methodological limitations include lack of randomisation (two studies were quasi-RCTs), failure to conceal allocation and inadequate methods for dealing with missing data. The overall evidence was based on studies of 'fair' methodological quality according to USPSTF.

The heterogeneity of control interventions might have influenced the pooled effect sizes found in this review. The authors adopted a pragmatic approach by combining different types of comparators, such as education, usual care and strengthening exercises. Including active comparators, such as strengthening exercises, might have attenuated the estimated effects associated with walking interventions. Future reviews should attempt to estimate the effect of walking interventions separately from either non-intervention control groups or non-exercise interventions on pain and function outcomes. This information may assist clinicians make treatment recommendations.

A range of walking programmes included in this review showed beneficial effects on pain and self-reported function among individuals with chronic musculoskeletal pain. However, at present, it is unclear whether specific characteristics of walking programmes, such as supervised programmes, homebased programmes or programmes using electronic feedback devices, are associated with greater effects sizes. Future studies investigating the influence of these characteristics on clinical outcomes are warranted.

\section{CLINICAL IMPLICATIONS}

This meta-analysis suggests that, in individuals with chronic musculoskeletal pain, walking interventions may improve pain 
at short and medium-term follow-up, and improve self-reported function at short, medium and long-term follow-up.

\section{Crystian B Oliveira, Rafael Z Pinto, Marcia R Franco}

Departamento de Fisioterapia, Faculdade de Ciências e Tecnologia, UNESP_Univ Estadual Paulista, Presidente Prudente, São Paulo, Brazil

Correspondence to Marcia R Franco, Rua Roberto Simonsen, 305, FCT/UNESP, Campus de Presidente Prudente, Bairro Jardim das Rosas, Presidente Prudente CEP: 19060-900, São Paulo, Brazil; marciarfranco@gmail.com

Contributors CBO, RZP and MRF selected the systematic review, interpreted the data and wrote the manuscript.

Competing interests None declared.

Provenance and peer review Not commissioned; externally peer reviewed.

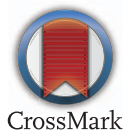

To cite Oliveira CB, Pinto RZ, Franco MR. Br I Sports Med 2016;50:1346-1347. Accepted 21 March 2016
Published Online First 13 April 2016

Br J Sports Med 2016;50:1346-1347. doi:10.1136/bjsports-2016-096245

\section{REFERENCES}

1 Reid KJ, Harker J, Bala MM, et al. Epidemiology of chronic non-cancer pain in Europe: narrative review of prevalence, pain treatments and pain impact. Curr Med Res Opin 2011:27:449-62.

2 Delitto A, George SZ, Van Dillen LR, et al. Low back pain. J Orthop Sports Phys Ther 2012;42:A1-57.

3 Zhang W, Nuki G, Moskowitz RW, et al. OARSI recommendations for the management of hip and knee osteoarthritis: part III: Changes in evidence following systematic cumulative update of research published through January 2009. Osteoarthr Cartil 2010;18:476-99.

4 Hootman JM, Macera CA, Ainsworth BE, et al. Association among physical activity level, cardiorespiratory fitness, and risk of musculoskeletal injury. Am J Epidemiol 2001;154:251-8.

5 Colbert LH, Hootman JM, Macera CA. Physical activity-related injuries in walkers and runners in the aerobics center longitudinal study. Clin J Sport Med 2000;10:259-63.

6 Eijsvogels T, George K, Shave R, et al. Effect of prolonged walking on cardiac troponin levels. Am J Cardiol 2010;105:267-72.

7 Tschentscher M, Niederseer D, Niebauer J. Health benefits of Nordic walking: a systematic review. Am J Prev Med 2013;44:76-84.

8 Hanson $S$, Jones $A$. Is there evidence that walking groups have health benefits? A systematic review and meta-analysis. Br J Sports Med 2015;49:710-15. 